Archives de sciences sociales des religions

127 | juillet - septembre 2004

Max Weber, la religion et la construction du social

\title{
Les fondements sociaux de l'agir normatif chez Durkheim et Weber : le rôle du sacré
}

Mauro Piras

\section{(2) OpenEdition}

1 Journals

Édition électronique

URL : http://journals.openedition.org/assr/1058

DOI : 10.4000/assr.1058

ISSN : $1777-5825$

Éditeur

Éditions de l'EHESS

Édition imprimée

Date de publication : 1 juillet 2004

Pagination : 139-166

ISBN : 2-222-96751-1

ISSN : 0335-5985

Référence électronique

Mauro Piras, "Les fondements sociaux de l'agir normatif chez Durkheim et Weber : le rôle du sacré » Archives de sciences sociales des religions [En ligne], 127 | juillet - septembre 2004, mis en ligne le 25 juin 2007, consulté le 30 avril 2019. URL : http://journals.openedition.org/assr/1058 ; DOI : 10.4000/ assr. 1058

(c) Archives de sciences sociales des religions 


\section{LES FONDEMENTS SOCIAUX DE L'AGIR NORMATIF CHEZ DURKHEIM ET WEBER : LE RÔLE DU SACRÉ}

Les pages qui suivent ont pour objet la thèse de la fondation sacrale des normes chez Durkheim et Weber. La visée d'une telle étude n'est toutefois pas seulement exégétique, mais aussi théorique. Elle est en effet la première étape d'une réflexion sur l'efficacité sociale des ordres normatifs. Le problème peut être formulé en ces termes : qu'est-ce qui fonde la validité effective des normes ? C'està-dire : quels sont les facteurs dont il faut tenir compte pour expliquer que, dans une société donnée, les acteurs sociaux assujettissent leur conduite à des ordres normatifs ? Deux éléments constituent la validité effective des normes : la force contraignante de celles-ci, qui s'imposent souvent contre les intérêts des acteurs mêmes, et leur force motivationnelle, qui pousse les acteurs à agir en vue de leur réalisation. La théorie sociale se doit de rendre compte de ces deux aspects, à travers la reconstruction du concept de norme et l'explication de son efficacité sociale.

Dans ce texte, on propose le premier moment de ce travail d'élucidation conceptuelle: l'exposition systématique de la théorie normative de Durkheim et Weber. Deux raisons justifient le choix de ces auteurs. D'abord, le rôle que la question normative occupe dans leurs recherches : pour Durkheim, cela va de soi, car toute sa réflexion est guidée par le projet d'une étude sociologique des « faits moraux ", et on trouve une définition et une redéfinition continue de ce domaine, dès la première édition de la Division du travail social (1893) jusqu'à l'ébauche inachevée d'Introduction à la morale (1917). Pour Weber, on connaît le rôle central du concept de "conduite de vie » (Lebensführung) à partir au moins de l'Éthique protestante (1904-1905) jusqu'aux autres études de Sociologie de la religion (1915-1920); mais, surtout, on trouve dans son essai sur Stammler (1907), dans les sections de sociologie du droit d'Économie et société (1912-1914), et dans les «Concepts sociologiques fondamentaux » (1919-1920) une élaboration théorique du concept de norme et de sa validité sociale. On a ainsi dans tous ces textes les premiers jalons d'une théorie normative. La deuxième raison est celle qui motive essentiellement cette étude : le rôle central de la sociologie de la religion dans leur œuvre, et l'analyse qu'on peut y trouver des rapports entre la validité factuelle des normes et la religion. Ici, en effet, on voudrait proposer comme 
approche à la question des normes la thèse durkheimienne du lien entre normes et sacré, en entendant cette thèse non tant comme l'établissement d'une filiation généalogique, mais plutôt comme une "parenté structurale » entre les deux phénomènes. Sur ce terrain la reconstruction systématique des thèses non seulement de Durkheim, mais aussi de Weber est d'une grande fécondité théorique. Il est certes vrai que chez Weber le concept de «sacré » n'est pas thématisé, ni mis au centre d'une théorie du lien social normatif. Toutefois, d'autres aspects de son travail sont pertinents pour discuter (et éventuellement critiquer) la thèse durkheimienne : d'un côté, l'analyse du rapport entre sacré, tradition et agir normatif; de l'autre, l'étude des différentes formes de "désenchantement du monde », qui permet de mieux cerner le sens des transformations historiques du sacré.

Dans cette première présentation, encore largement incomplète, on procèdera comme suit : 1) d'abord, on exposera la définition durkheimienne du " fait moral », sa thèse sur la fondation sacrale des normes, et sa théorie du sacré, et on évaluera brièvement la portée théorique de ces positions ; 2) ensuite, on analysera le concept de norme chez Weber, sa théorie sur les fondements de validité sociale des normes, et quelques points de sa sociologie des religions qui peuvent aider à éclairer le problème ; 3) enfin, on essaiera de formuler quelques remarques conclusives, forcément provisoires, vu l'état de la recherche.

\section{Durkheim : de la norme au sacré}

Durkheim (1) s'est occupé pendant toute sa carrière intellectuelle des «faits moraux » : il a créé cette expression précisément pour délimiter le comportement normatif d'un point de vue sociologique. C'est pourquoi on trouve dès ses premiers écrits une réflexion théorique sur ce domaine et, dans les années 1890, des tentatives de mise au point générale. Les textes les plus importants de cette période pour la définition du concept de norme sont les suivants : la première "Introduction » à la Division du travail social, de 1893, qui contient une trentaine de pages de "philosophie morale », qui manquent dans les éditions ultérieures (1902) (2); la Division du travail social, dans les parties concernant la morale et le droit, dont le texte est fondamentalement inchangé dans les éditions successives; le cours Leçon

(1) Abréviations utilisées pour la citation des œuvres de Durkheim :

DTS 93: De la division du travail social (1ère éd.), Paris, Alcan, 1893 ;

DTS : De la division du travail social (2ème éd.), Paris, Alcan, 1902 ;

EM: L'éducation morale, Paris, PUF, 1963 ;

FE : Les formes élémentaires de la vie religieuse, Paris, Alcan, 1912 ;

JS : Journal sociologique, Paris, PUF, 1969 ;

LS : Leçon de sociologie : physique des mours et du droit, Paris, PUF, 1969 ;

SP : Sociologie et philosophie, Paris, Alcan, 1924 ;

SSA : La science sociale et l'action, Paris, PUF, 1970 ;

Textes 1, 2, 3: Textes, vol. 1, 2, 3, Paris, Minuit, 1975.

(2) DTS 93, pp. 4-39 ; cf. aussi Textes 2, pp. 257-288. Sur cette première "Introduction », cf. François A. IsAmbert, «Durkheim : une science de la morale pour une morale laïque », Archives de sciences sociales des religions, 35, 1990, pp. 129-146: pp. 132-134. 
de sociologie : physique des mours et du droit, que l'on peut évaluer être de 18981900. Dans ces travaux, la norme est définie essentiellement par l'obligation et la sanction. Toutefois, à partir de la fin des années 1890, Durkheim retravaille sa conception des « faits moraux », après le «tournant» de 1895, qui marque la naissance de son intérêt pour la sociologie des religions et l'intuition du lien nécessaire entre sacré et normes sociales (3). Les résultats de cette révision apparaissent dans les textes les plus marquants écrits entre 1902 et 1917 : L'éducation morale (19021903), la « Détermination du fait moral » (1906), « Jugements de valeurs et jugements de réalité » (1911) (4) et Les formes élémentaires de la vie religieuse (1912). À ceux-ci on peut ajouter «Le dualisme de la nature humaine et ses conditions sociales » (1914) (5), et le fragment d' "Introduction à la morale » (1917) (6), rédigé par Durkheim juste avant sa mort et publié posthume. Dans ce deuxième groupe de textes, le fait moral est défini, comme il est bien connu, par deux aspects contradictoires, l' « obligation » et la « désirabilité », qui imposent au théoricien la tâche difficile de penser leur relation réciproque.

\subsection{Le concept de norme sociale}

Durkheim part de la distinction (kantienne) entre règles techniques et règles morales. La règle est entendue comme un principe qui impose de suivre une certaine conduite ; à l'intérieur de cette classe générale des "règles de conduite », les «faits moraux » constituent un sous-ensemble, parce que toutes les règles de conduite ne sont pas morales (7). Pour les définir par rapport aux autres, il faut considérer quelles sont les conséquences de leur violation : lorsque je ne respecte pas une règle technique (par exemple, un principe économique, médical, de production, etc.) mon action risque simplement de ne pas réussir, d'échouer (8). Lorsqu'au contraire je ne respecte pas une règle morale, je provoque une réaction de réprobation et de blâme de la part de la société, qui s'oppose à cet acte : "Celui qui a commis un meurtre ou un vol, par exemple, est puni d'une peine matérielle ; celui qui déroge aux lois de l'honneur encourt le mépris public ; celui qui a manqué aux engagements librement contractés est obligé de réparer le dommage qu'il a causé, etc. » (9). Contrairement au premier type de règle, il y a, en cas d'infraction, une réaction sociale, qui se manifeste par la «sanction », ce qui permet d'affirmer que « tout fait moral consiste dans une règle de conduite sanctionnée » (10).

«L'élément essentiel de toute règle morale» est donc, dans les textes écrits entre 1893 et 1900, la sanction (11). Cela ne veut pas dire que la sanction constitue

(3) Steven Lukes, Émile Durkheim. His Life and Work, Stanford (Cal.), Stanford University Press, 1985, pp. 237-238; Jeffrey C. AleXAnder, Structure and Meaning, New York, Columbia University Press, 1989, pp. 138-139.

(4) $S P$, pp. 49-90, et pp. 117-141.

(5) SSA, pp. 314-332.

(6) Textes 2, pp. 313-331.

(7) DTS 93, p. 23.

(8) DTS 93, p. $23 ; L S$, p. 42

(9) DTS 93, p. 23.

(10) DTS 93, p. 24 (souligné par moi).

(11) $L S$, p. 42. 
le fait moral, elle n'est que le signe extérieur, «objectivé », du vrai fondement constitutif de la norme, son caractère obligatoire : « il n'y a pas de règle [morale] là où il n'y a pas d'obligation » (12). D'où vient à son tour le sentiment d'obligation qui fonde la sanction? Du rapport entre la règle et la sanction elle-même. La sanction n'est pas une conséquence de «l'acte pris en lui-même, mais de ce qu'il est conforme ou non à une règle de conduite préétablie», qui «le permet ou le prohibe »(13). Cette règle est ce qui détermine le caractère obligatoire des normes : les faits moraux sont des « règles impératives » (14). Mais ce caractère obligatoire ne peut pas agir s'il n'est pas senti comme un devoir transcendant de la part des acteurs sociaux qui, en appliquant les sanctions, le manifestent. On sait en effet que Durkheim définit la «conscience collective» comme l'ensemble des « croyances et sentiments communs » aux membres d'une société ; il est évident que les faits moraux sont un élément fondamental de la conscience collective (il suffit de rappeler que, pour Durkheim, les « règles qui prohibent [les] actes [criminels] sont gravées dans toutes les consciences » (15)). L'obligation, donc, n'est pas un fait purement extérieur, mais passe par les consciences individuelles : elle est le sentiment qu' on ne peut enfreindre une norme parce qu'elle est « valable objectivement», " transcendante » par rapport au sujet de l'action (16). C'est pourquoi Durkheim peut conclure que ce qui détermine le caractère moral ou immoral d'un acte - donc : ce qui constitue les faits moraux - est la conscience collective, ce sont les « sentiments collectifs» $(17)$.

On peut résumer ainsi le concept de norme qui émerge des textes de Durkheim rédigés entre 1893 et 1900 : la norme est une règle de conduite socialement sanctionnée, engendrée par une «conscience collective », c'est-à-dire par la croyance, partagée par la moyenne des membres d'une société, que cette norme est obligatoire. On voit donc que pour Durkheim l'acceptation commune de la norme est constitutive de celle-ci ; toutefois, les éléments qu'on a analysés jusqu'ici ne disent encore rien sur les fondements de fait de cette acceptation. Pour aborder cet aspect, on peut aussi prendre en considération les textes de la deuxième période d'élaboration du concept de norme sociale. Dans cette deuxième période, on remarque trois différences importantes par rapport à la première : 1) une distinction conceptuellement plus claire entre règles techniques et règles morales; 2) la définition de la norme non seulement par l' " obligation », mais aussi par la "désirabilité »; 3) la thèse de la fondation sacrale des normes, qui, déjà présente dans les textes précédents, se trouve développée et mise en évidence. On rappellera les points 1) et 2), pour montrer comment le concept général de norme s'enrichit, sans toutefois entrer en contradiction avec le cadre conceptuel introduit dès 1893. Ensuite, on examinera le rapport entre lien normatif et sacré, comme réponse à la question de la fondation sociale des normes.

En ce qui concerne la distinction entre règles techniques et règles morales, qui encore dans Physique des mours (1898-1900) n'était pas très bien tracée,

(12) DTS 93, p. 30 ; cf. aussi p. 28.

(13) $L S$, p. 42.

(14) DTS 93, p. 28.

(15) DTS, p. 40.

(16) DTS, pp. $39-40 ; 46-48 ; 74-78 ; 207-208$.

(17) DTS, pp. 47-48. 
Durkheim arrive en 1906 à une formulation conceptuelle très efficace, en s'appuyant encore une fois sur une distinction kantienne : il introduit en effet le couple analytique-synthétique pour définir le rapport entre la norme et les conséquences qui découlent de sa violation. Lorsqu'on enfreint une règle technique, les conséquences de cette violation se produisent "mécaniquement», parce qu'il y a un lien matériel direct entre l'acte et ses conséquences. C'est pourquoi, en forçant un peu la terminologie kantienne, Durkheim décrit ce lien comme «analytique » (18). Pour les normes morales, au contraire, le lien est « synthétique » : les caractères de l'acte ne contiennent aucunement les conséquences sociales qui en découlent, ce qui est bien prouvé par le fait que les sanctions changent selon les milieux sociaux, et que la distinction même entre actes sanctionnés ou non varie. Le lien est synthétique parce que la sanction est « ajoutée de l'extérieur » à l'acte ; ce qui crée cette « unification » est la règle acceptée dans une société : "La sanction est une conséquence de l'acte qui ne résulte pas du contenu de l'acte, mais de ce que l'acte n'est pas conforme à une règle préétablie » (19). On voit bien par cette citation que la distinction conceptuelle est la même que pour la première période, mais qu'elle a gagné en clarté par l'utilisation (quoiqu'un peu abusive) du couple kantien analytique-synthétique.

Le second point, c'est-à-dire la définition de la norme non seulement par le « devoir », mais aussi par le « désir du bien », est aussi très connu; il mérite qu'on s'y arrête quelques instants en raison de sa fonction dans l'établissement d'une analogie structurale entre normes et sacré. La "désirabilité », comme deuxième élément constitutif du fait moral, apparaît dans les écrits de Durkheim à partir des années 1901-1902 : on en trouve la trace dans un compte rendu de 1901 (20), et un premier développement dans l'Éducation morale, cours tenu en 1902-1903 (21). La formulation publique devenue désormais classique se trouve dans la « Définition du fait moral » (1906). Ici, après avoir commenté le caractère obligatoire des normes, Durkheim critique la position kantienne, qui limite le fait moral à ce seul aspect; selon Durkheim, l'eudémonisme doit au contraire entrer nécessairement dans l'agir moral. La raison fondamentale de cette thèse se trouve dans le problème de la motivation: il est impossible que les hommes agissent en vue d'une fin morale seulement en raison du sentiment d'obligation. Pour que la conduite morale soit effective, il faut qu'il y ait un "ressort psychologique »; la fin morale est aussi «bonne ", elle est un bien vers lequel on tend, parce qu' on s'y reconnaît, elle est donc «désirable»(22). Seulement, dit Durkheim, cette désirabilité participe en quelque mesure aussi du caractère de l'obligation : même lorsqu'on accomplit une action suivant un élan moral, cette action comporte un effort sur l'individualité du sujet, qui «sacrifie» quelque chose pour l'accomplir(23). C'est pourquoi Durkheim insiste sur la réciprocité et l'absence de priorité entre les deux éléments : il y a nécessairement obligation, parce qu'autrement il n'y aurait pas de fait moral ; mais en même temps il y a « désir du bien », parce qu'autrement il n'y aurait pas de

(18) $S P$, pp. 60-61.

(19) $S P$, pp. 61-62.

(20) Cf. « Genèse des idées morales » (1901), Textes 2, pp. 332-333.

(21) $E M$, pp. 57 sq.

(22) $S P$, pp. 63-64.

(23) $S P$, p. 64. 
force motivationnelle pour accomplir le devoir (24). Le concept qui permet de comprendre cette coexistence est celui d'autorité morale, qui est digne de respect et qui s'impose par son prestige (25).

Quelle est la signification théorique de cet enrichissement du concept de norme ? Premièrement, comme on l'a déjà souligné, on dispose maintenant d'un outil conceptuel pour penser la force motivationnelle des normes sociales, qui échappe à toute conceptualisation restreinte à l'aspect de la contrainte : l'aspect affectif, non intellectuel, de l'adhésion culturelle aux normes est un facteur historique qu'on ne peut pas négliger. Deuxièmement, l'introduction du «désir du bien » permet de rendre compte d'une expérience singulière, propre à l'acceptation des normes, que Durkheim cherche à articuler par l'idée d'une " coexistence » nécessaire de l'obligation et du désir : c'est l'expérience de la «transcendance de soi » qui s'accomplit dans la réalisation de l'acte moral (26). Dans la reconnaissance d'un impératif moral, en effet, le sujet se reconnaît dans une réalité symbolique « objective », parfaitement transcendante par rapport à son individualité, mais dans laquelle, à la fois, il réalise sa propre existence comme personne morale : dans l'adhésion morale le sujet appartient simultanément à soi et à quelque chose qui lui est extérieur (27). Ces deux « ouvertures théoriques » introduites par l'idée du «désir» permettront ensuite à Durkheim de lier l'agir normatif et le sacré, non seulement d'un point de vue génétique, mais aussi dans une perspective, pour ainsi dire, "phénoménologique ».

\subsection{L'efficacité sociale des normes : le sacré}

Jusqu'ici, on a exposé la structure du concept de norme chez Durkheim, dont on a vu l'enrichissement dans les textes de la période d'après 1901 . On peut maintenant passer au niveau suivant : l'explication de l'efficacité sociale des normes. La réponse de Durkheim est connue : la force à la fois contraignante et motivationnelle des normes dérive de leur origine dans le « sacré », et se maintient dans les sociétés laïcisées grâce à ce caractère sacral des fondements normatifs. Cette thèse est justifiée par Durkheim de deux manières, l'une « génétique », et l'autre «structuraleanalogique » (qui ébauche une sorte de phénoménologie parallèle du sacré et de la norme). Le premier type d'explication ne fait que rappeler une évidence historique: la genèse de tous les ordres normatifs dans les religions, le caractère originellement religieux du droit pénal, la prééminence originelle du droit religieux, etc. (28). Le deuxième type d'explication, dont quelques éléments apparaissent déjà dans la Division du travail social (29), se développe progressivement après le début des recherches de sociologie religieuse (1895) et trouve sa définition dans les textes les plus importants écrits entre 1906 («Détermination du

(24) $S P$, pp. 64-65; 67.

(25) $S P$, p. 67.

(26) Cf. Hans Joas, Die Entstehung der Werte, Frankfurt a. M., Suhrkamp, 1999, pp. 103 sq.

(27) Cf. Jürgen Habermas, Theorie des kommunikativen Handelns. II, Frankfurt a. M., Suhrkamp, 1981, p. 83.

(28) DTS, pp. 108 sq. ; 119 sq.

(29) Cf. par exemple les considérations sur le sacré dans les sociétés modernes et sur la « religion de l'individu »: DTS, p. 147. 
fait moral ») et 1912 (Les formes élémentaires de la vie religieuse). Déjà dans l'étude sur l'inceste (1896-1897) Durkheim ébauche un parallélisme entre l'horreur morale provoquée par l'idée de l'inceste et l'horreur provoquée, dans une culture primitive, par l'idée «d'un mélange possible entre ce qui est tabou et ce qui est profane » (30). Dans l'étude " De la définition des phénomènes religieux » (18971898) on trouve la thèse selon laquelle la société est la source aussi bien du partage sacré/profane que du partage moral/immoral (31). On va maintenant suivre l'articulation de ces éléments dans les Formes élémentaires de la vie religieuse (et en partie également dans la «Détermination du fait moral ») pour tracer une première esquisse de la théorie durkheimienne de la validité sociale des normes.

Pour Durkheim, l'opposition entre le sacré et le profane est constitutive de toute religion et, dans un certain sens, de tout ordre normatif. Elle engendre une division de la réalité entre deux règnes radicalement différents l'un de l'autre, qui ne peuvent communiquer entre eux qu'au moyen de cérémonies et de personnes exceptionnelles; la vie pratique elle-même est partagée selon cet ordre, qui sépare les actions «quotidiennes », « ordinaires» de la reproduction sociale matérielle, des actions rituelles, sacrées (32). Le sacré est tout ce à quoi on doit un respect particulier, absolu; il ne doit être violé d'aucune façon, parce que son caractère propre est précisément une dignité supérieure, qui lui assujettit toutes les autres réalités. Ce qui est sacré ne peut jamais être « utilisé » pour des fins pratiques, il ne peut jamais être considéré comme une réalité quelconque ; il ne peut être approché, touché, nommé, etc., que par des rites et des cérémonies spéciales. Le passage du profane au sacré est en principe impossible, sauf à travers ces cérémonies, qui peuvent amener à une sorte de "conversion", à une transformation "totius substantiae » de la chose ou de la personne (33). Le sacré se manifeste d'abord dans des « objets sacrés »: ceux-ci font l'objet des célébrations, des actions rituelles, etc. Toutefois, il ne s'y épuise pas : il s'agit plutôt d'une qualité générale, indéterminée, qui peut «se poser sur tout»; ici, comme il est bien connu, Durkheim reprend à son compte la notion de mana, élaborée en particulier, par Mauss. Le mana aussi, comme en général le sacré, exerce une double action, physique et morale (34). Le sacré est l'objet auquel s'adressent les pratiques rituelles, il est le centre des moments de vénération et de célébration collective à travers lesquels se produit la solidarité du groupe autour de ses propres croyances (35).

Le sacré est donc une autorité indiscutée, indiscutable, qui, échappant au traitement ordinaire réservé aux choses communes, échappe à l'objectivation conceptuelle et linguistique ; par conséquent, il agit toujours de manière irréfléchie, s'imposant sans médiation à tous ceux qui pratiquent une religion donnée. Sa force contraignante se manifeste de deux manières: d'un côté, à travers le caractère terrible du châtiment pour le sacrilège ; de l'autre, en vertu de l'autorité transcendante, divine, digne de respect en soi, du sacré lui-même (36). Les deux moments

(30) «La prohibition de l'inceste et ses origines » (1896-1897), JS, pp. 37-101: p. 91.

(31) «De la définition des phénomènes religieux » (1897-1898), JS, pp. 140-165: pp. 161-163.

(32) FE, pp. $50-54 ; 165$.

(33) $F E$, p. 54

(34) FE, pp. $277-290$

(35) $F E$, p. $56 ; 299-301$.

(36) FE, p. 271. 
sont indissociables, en sorte que l'impératif religieux s'affirme comme valable par le seul fait de son existence. Il est une force objective qui s'impose à l'individu à la fois de l'extérieur et de l'intérieur (37). Le sacré est une objectivité transcendante mais, dans son rapport avec lui, l'individu retrouve soi-même, sa propre identité, dans quelque chose qui lui est extérieur. Dans le sacré se réalise une expérience de « transcendance de soi » (38).

De l'ensemble de toutes ces caractérisations du sacré dérive la division entre sacré et profane; le premier des deux délimite négativement comme profane le reste de la réalité, y projetant toute la sphère de l' « individuel » : depuis l'existence strictement matérielle, aux activités économiques, aux pratiques quotidiennes nécessaires à l'organisation de la vie sociale. Dans le sacré, au contraire, converge tout ce qui concerne l'identité collective de la communauté : ce qui est sacré est commun, c'est ce qui forme les consciences individuelles selon des normes de comportement partagées, ce qui délimite le groupe des autres groupes, ce qui crée et vivifie les rapports sociaux (39). Ce caractère social est exprimé par la nature impérative du lien sacral, qui s'impose comme une tierce force autonome, entre la conscience individuelle et le monde extérieur.

L'analyse du sacré (40) fournit à Durkheim la réponse au problème de l'« efficacité sociale des normes ». La structure du sacré est parallèle à celle de la norme : il exerce une force sociale aussi bien par la crainte des peines infligées au sacrilège que par l'autorité morale dont la dignité du sacré est investie (41). En outre, dans le sacré s'enracine cette expérience de «transcendance du soi » qui, dans la norme aussi, permet au sujet agissant de se reconnaître dans une autorité extérieure à sa propre volonté (jusqu'au point qu'elle puisse s'opposer à ses pulsions et à ses intérêts), mais constitutive de son identité. Tant dans le sacré que dans la norme, l'efficacité dérive essentiellement de cette expérience, qui fusionne le respect pour ce qui est «inviolable » avec la crainte d'une autorité omnipotente. Le sacré est, comme la norme socialement efficace, une force "indiscutable », envers laquelle l'idée même de la critique, de la confrontation avec la réalité empirique, apparaît comme un sacrilège.

Cette position durkheimienne représente certes une première réponse théoriquement féconde au problème de la validité de fait des normes. Toutefois, si on veut étendre de façon pertinente cette analyse du sacré aux normes sociales, il faut remarquer que tout cela est valable pour les ordres normatifs des sociétés traditionnelles, où résistent plusieurs domaines du comportement qui échappent à la critique discursive et où les règles transmises conservent, par cela même, une aura de sacralité. Ce niveau correspond à la conscience morale que Kohlberg appelle

(37) Jürgen Habermas, Theorie des kommunikativen Handelns. II, op. cit., pp. 78-79; 83.

(38) Hans JoAs, Die Entstehung der Werte, op. cit., pp. 94-95.

(39) FE, p. 165.

(40) Je me suis limité à reconstruire les thèses de Durkheim du point de vue qui nous intéresse ici ; il n'est pas possible, dans cette première ébauche d'analyse, d'entrer dans toutes les difficultés posées par le concept durkheimien de sacré. Pour les critiques adressées à sa théorie, cf. au moins François A. IsAmbert, Le sens du sacré, Paris, Minuit, 1982, III Partie, et Danièle Hervieu-LÉGer, La religion pour mémoire, Paris, Cerf, 1993, chap. III ; cf. aussi, dans une perspective différente, mais aboutissant à des critiques semblables, Carsten ColPE, Über das Heilige, Frankfurt a. M., Hain, 1990.

(41) $S P$, pp. 68-70. 
« conventionnelle», dans laquelle le sujet applique, même réflexivement, les normes apprises dans sa socialisation, mais il n'en remet pas en question le contenu sémantique (42). Toujours selon Kohlberg, pour la plupart des personnes, même dans les sociétés modernes, la couche fondamentale des convictions normatives se situe à ce niveau (43). Avec ces spécifications, on peut partir de l'hypothèse que le fondement de la validité factuelle des normes soit précisément cette «conviction irréfléchie », " athématique », « indiscutable », dont la source est sans doute de nature religieuse.

Le problème théorique qui s'ouvre ici concerne l'origine et la nature de cette force morale religieuse. C'est sur ce terrain, en effet, qu'il est possible de porter au jour la structure spécifique du lien normatif. On connaît la réponse donnée par Durkheim à cette question : le sacré n'est que le produit de l'ordre social lui-même, il est dans un certain sens la projection du pouvoir que la société exerce sur les individus (44). Il n'est pas nécessaire ici d'entrer dans les détails de cette thèse controversée, que Durkheim décline de différentes manières, et qu'on ne peut donc réfuter seulement par les arguments de la circularité logique ou par le refus du fonctionnalisme sociologique (45). Il y a toutefois un point de l'analyse durkheimienne qui mérite d'être pris en compte directement, parce qu'il identifie une source possible de ces certitudes irréfléchies qui seules peuvent stabiliser l'agir normatif. Durkheim remarque que l'autorité morale a son origine dans l' « opinion », qu'elle est un « état de l'opinion ». L'adhésion à un principe moral, de la part de l'individu, est renforcée par la reconnaissance donnée à cette adhésion de la part des autres: le fait que les membres du groupe partagent les mêmes convictions engendre la norme. Cela a déjà lieu dans la vie sociale ordinaire : l'identité morale du moi est constituée par la reconnaissance que le milieu social accorde aux actions du sujet, sujet qui, en revanche, n'est pas sûr de son intégrité et de la validité de ces actions lorsque ce consensus est nié (46). Cet enracinement de la validité normative dans la reconnaissance réciproque est accentué dans les moments fondateurs des valeurs morales : les moments d' "effervescence collective » dans lesquels, à travers les rites, les célébrations, les fêtes, etc., la communauté renouvelle son identité collective, ravive le contenu de ses normes morales (47). On régénère l'appartenance au même groupe « moral ». Les mouvements d'effervescence collective constituent les individus en membres du groupe, les poussant à transcender leur individualité dans la norme morale; ces actions rituelles deviennent ainsi les symboles de l'identité collective, qui la préservent et la stabilisent (48).

Cette hypothèse libère l'origine du phénomène normatif du seul contexte religieux, l'enracinant dans un mécanisme social plus large, valable aussi pour la

(42) Lawrence Kohlberg, Essays in Moral Development. II, San Francisco-Cambridge-New York, Harper \& Row, 1984, p. 58.

(43) Ibid., p. 57.

(44) FE, pp. 302-304, et tout le chapitre VII du Livre II.

(45) Pour ce type de critiques adressées à la thèse de Durkheim, cf. Steven Lukes, Émile Durkheim..., op. cit., pp. 480 sq.

(46) FE, pp. 297-299.

(47) FE, pp. 299-301; 308-310;313-314.

(48) FE, pp. 330-331. 
morale laïque. Par ailleurs, on peut aussi la soutenir sans accepter la théorie « fonctionnaliste » de Durkheim sur la connexion entre religion et société. La force sociale du lien normatif peut au contraire, à travers une révision de la théorie des " effervescences collectives », être ramenée à une "dialectique de la reconnaissance » (49).

\subsection{Portée et limites de la position durkheimienne}

On peut tirer un premier bilan - tout à fait provisoire - de la conception durkheimienne des normes et de leur validité sociale. D'abord, les éléments féconds du point de vue théorique, parmi lesquels il faut distinguer le niveau de l'explicitation conceptuelle de celui des thèses explicatives. En ce qui concerne le premier, Durkheim élabore un cadre conceptuel général qui, sous le double aspect de l'obligation et du désir, permet d'intégrer dans l'analyse des aspects importants comme la force motivationnelle des normes et l'expérience de transcendance de soi liée (au moins en partie) à l'agir normatif. En ce qui concerne les thèses explicatives, la filiation sacré-normes permet de rendre compte du caractère irréfléchi, non-objectivable et donc fondateur de valeurs et de hiérarchies conceptuelles, qui est propre au noyau de l'agir normatif (50); en outre, la thèse qui place les " états de l'opinion » et les «effervescences collectives» à la source des valeurs et des normes peut être utile pour éclairer en général la formation des identités sociales (51).

Quelles sont toutefois les limites, déjà visibles ici, de cette approche ? Sur le plan des «concepts fondamentaux», Durkheim reste trop lié au seul aspect de l'«adhésion culturelle» aux normes; quoiqu'il souligne occasionnellement que l'obéissance de fait aux normes est influencée par de nombreux facteurs (52), il n'analyse pas ces facteurs (l'intérêt, l'acceptation pragmatique, la tradition, la domination, etc.), ni surtout, le rapport qu'il faut établir entre ceux-ci d'un côté et la reconnaissance de la valeur "sacrée" des normes de l'autre. Toutefois, le problème théorique se trouve précisément ici : si le caractère «indisponible » et « non objectivable » du noyau normatif constitue l'agir normatif, comment peut-on penser la présence et l'articulation d'autres facteurs causaux ? En ce qui concerne le plan des thèses explicatives, il faut remarquer qu'elles souffrent des limites conceptuelles de la philosophie du sujet : certes, Durkheim montre bien le caractère trans-subjectif, social, des « faits moraux », mais, puisque son ontologie fondamentale se réduit néanmoins aux rapports entre le sujet et l'objet, il doit trouver une explication de l'intersubjectivité morale qui ait comme seuls éléments de base la conscience et ses représentations. C'est pourquoi il doit recourir à la psychologie des foules: la seule manière d'expliquer la possibilité que le sujet sorte de soi-

(49) Cf. Axel Honneth, Kampf um Anerkennung, Frankfurt a. M., Suhrkamp, 1992 ; cf. aussi Axel HonNETH, « Anerkennung und moralische Verpflichtung », Zeitschrift für philosophische Forschung, 51, 1997, pp. 25-41.

(50) Cf. Jeffrey C. AleXAnder, Action and its Environments, New York, Columbia University Press, 1988, pp. 319-322.

(51) Cf. Hans JoAs, Die Entstehung der Werte, op. cit.

(52) Cf., par exemple, «Introduction à la morale» (1917), Textes 2, p. 330. 
même et s'identifie avec un sujet collectif, sans forcer les limites du rapport sujetobjet, est de supposer que dans des moments exceptionnels d' " effervescence » (proches de l'extase) les limites des consciences individuelles soient brisées. Seule la mécanique affective peut fonder, pour Durkheim, une "communication des consciences », autrement condamnées à rester fermées sur elles-mêmes. Ce qui manque pour éviter le recours nécessaire à la psychologie des foules, et pour réintégrer de manière théoriquement féconde ces thèses, est l'idée de la fondation originellement intersubjective, à travers la socialisation communicationnelle, des significations et ainsi des normes.

\section{Weber : une conception "évolutive" du sacré}

Comme Durkheim, Weber (53) aussi, dans son effort de fondation de la sociologie, vise à délimiter l'étude empirique des valeurs de leur discussion éthique ou juridique (54). Les bases théoriques d'une telle étude sont posées surtout dans les textes de méthodologie et de théorie générale, où Weber élabore le concept de sens « intentionné » ou " subjectif» (gemeinte/subjektive Sinn), c'est-à-dire du « sens visé par les acteurs », considéré dans son efficacité empirique, qui se distingue clairement du "sens (métaphysiquement) objectif», considéré comme objet d'une réflexion visant à évaluer sa validité idéelle (55). Sur le terrain spécifique des normes, Weber consacre beaucoup de pages à bien délimiter réciproquement l'étude "sociologique » et l'étude " dogmatique » du droit, notamment dans quelques textes fondamentaux comme la critique de Stammler («R. Stammler et le 'dépassement' de la conception matérialiste de l'histoire », 1907), les sections "L'économie et les ordres sociaux » (1912) et "Sociologie du droit » (1914) dans Économie et société, et les « Concepts sociologiques fondamentaux » (1919-1920), toujours dans Économie et société (56).

(53) Abréviations utilisées pour la citation des œuvres de Weber : RelS $(W u G)$ : «Religionssoziologie » (1914), in $W u G$, pp. 245-381; RechtS $(W u G)$ : «Rechtssoziologie » (1914) in $W u G$, pp. 387-513 ;

RS I : Gesammelte Aufsätze zur Religionssoziologie, I, Tübingen, Mohr, 1920 ;

SG $(W u G)$ : «Soziologische Grundbegriffe » (1919-1920), in WuG, pp. 1-30.

SS : Gesammelte Aufsätze zur Soziologie und Sozialpolitik, Tübingen, Mohr, 1924 ;

TdH $(W u G):$ «Die Typen der Herrschaft» (1919-1920), in WuG, pp. 122-176;

$\mathrm{WgO}(W u G)$ : «Die Wirtschaft und die gesellschaftlichen Ordnungen » (1912), in $W u G$, pp. 181-198;

WL: Gesammelte Aufsätze zur Wissenschaftslehre, Tübingen, Mohr, 1985 ;

WuG: Wirtschaft und Gesellschaft. Grundriss der verstehenden Soziologie, Tübingen, Mohr, 1972.

Les traductions françaises des citations sont tirées d'Économie et société, Paris, Plon, 1971, qui contient les renvois à la pagination de l'édition allemande citée. Pour les autres ouvrages, c'est moi qui traduis.

(54) Ceci est en effet, par exemple, le sens du célèbre débat sur la "neutralité axiologique » des sciences sociales : cf. «Der Sinn der 'Wertfreiheit' der soziologischen und ökonomischen Wissenschaften » (1918), WL, pp. 489-540.

(55) Cf. SG $(W u G)$, pp. $1-2 ; 13$.

(56) «R. Stammlers 'Überwindung' der materialistischen Geschichtsauffassung » (1907), WL, pp. 291-359, avec le «Nachtrag», WL, pp. 360-383; "Die Wirtschaft und die gesellschaftlichen Ordnungen » (1912), WuG, pp. 181-198; «Rechtssoziologie » (1914), WuG, pp. 387-513 ; « Soziologische Grundbegriffe » (1919-1920), WuG, pp. 1-30. 
Le résultat de ce long effort théorique est la construction d'un concept sociologique complexe d' " agir normatif», dans lequel, à la différence de Durkheim, les nombreux facteurs qui peuvent le déterminer de fait (l'acceptation culturelle de la norme, la tradition, la pression du milieu, l'intérêt, l'acceptation pragmatique, etc.) sont articulés conceptuellement, laissant ouvert un espace théorique pour diverses explications de l'agir (au cas par cas) et pour une confrontation toujours possible entre la norme et l'agir concret. La théorie de la validité factuelle des normes chez Weber est donc fondée sur la «composition» de facteurs «multiples ». Il n'y a cependant pas, dans cette théorie, une connexion directe avec l'analyse des phénomènes religieux; simultanément, il est évident qu'elle possède une fonction heuristique importante précisément pour l'analyse des conduites de vie religieuses et de leur influence sur les autres sphères de l'agir social. C'est pourquoi il est légitime d'interroger la Sociologie de la religion pour pousser plus loin la théorie normative. On verra ainsi que, déjà dans le cadre théorique général, on peut repérer parmi les facteurs stabilisateurs de l'agir normatif le " caractère sacré de la tradition » et la « sacralisation des conduites habituelles »; mais, surtout, il est possible de mettre en lumière une "évolution" du lien normatif, car Weber montre que celui-ci n'émerge qu'à un stade donné de l'histoire religieuse, qu'il se renforce seulement dans certaines conditions et qu'inversement l'emprise du normatifreligieux diminue à mesure qu'augmente l'« objectivation » du monde social.

\subsection{La construction du concept de norme}

Comme pour Durkheim, on peut partir de la distinction kantienne entre règles techniques et règles morales; il est d'ailleurs remarquable que non seulement dans la construction d'une théorie normative, mais dans l'architecture générale de leur théorie sociale aussi bien Durkheim que Weber s'appuient consciemment sur des dichotomies kantiennes, dont celle entre causalité naturelle et règne des fins se répète, pour le premier, dans la dichotomie profane/sacré, agir technique/agir normatif, et, pour le second, dans la dichotomie zweckrationales/wertrationales Handeln (57). Du point de vue spécifique de l'agir normatif, l'articulation de cette dichotomie chez Weber se trouve dans son étude critique sur Stammler. Weber y analyse le concept de "règle », à fin de déterminer le rôle des règles juridiques dans la vie sociale. Dans cette analyse émergent trois significations du concept. La première ne nous intéresse pas ici, parce qu'il s'agit de ce que Weber appelle " règles de l'expérience » (Erfahrungsregeln) : ce sont des régularités empiriques, que l'on peut repérer dans la réalité (Regelmäßigkeiten des Geschehens), et qui peuvent être aussi bien des connexions causales nécessaires, que des règles valables avec une bonne probabilité statistique, que, enfin, des règles empiriques sans exception, mais qui n'ont pas de nécessité scientifique (58). Mis à part ce premier type, on rencontre les « règles de conduite » qui, selon la terminologie de l'étude sur Stammler, s'appellent Zweck-Maximen et Norm-Maximen. Les Zweck-Maximen sont les règles techniques en général, aussi bien celles de production d'un objet,

(57) Cf. à ce sujet les remarques très intéressantes de Wolfgang Schluchter, Religion und Lebensführung. I, Frankfurt a. M., Suhrkamp, 1988, pp. 206-208.

(58) Cf. $W L$, p. 323. 
que les règles économiques, les règles hygiéniques, etc. (59). Elles sont des règles de l'agir " conforme à une fin » (zweckmäßiges Handeln) (60), qui prescrivent les moyens les plus adéquats pour réaliser une fin désirée. Elles ont donc la structure du raisonnement pratique : "si tu veux $\mathrm{X}$, tu dois accomplir $\mathrm{Y}$ ». C'est pour cette raison qu'elles sont fondées sur le savoir nomologique exprimé par les « règles de l'expérience »: le raisonnement pratique n'est autre que l'inversion de l'énoncé cognitif, nomologique, "à l'événement $Y$ suit régulièrement l'événement $\mathrm{X}$ » (61). Ces règles ne sont donc pas de nature sociale, parce que leur contenu sémantique est déterminé par la seule connaissance de l'objet, et que leur caractère impératif ne dérive que des fins posées par le sujet et du désir de les réaliser au moindre coût (62). Elles ont néanmoins, comme toute règle de conduite, un caractère évaluatif, puisque la relation moyens-fins établie grâce à la connaissance empirique devient un standard d'évaluation (Wertungsstandard) pour juger le processus réel ; évidemment, ce standard n'est pas moral, mais technique, concernant l'efficacité des choix par rapport aux fins (63). Weber n'explicite pas, comme marque qualifiante de ce type de règles, quelles sont les conséquences qui suivent de leur transgression, mais il est évident par le dernier point cité qu'elles se résument dans l'insuccès.

Les Norm-Maximen sont les règles pratiques, construites symétriquement aux règles techniques dans l'étude sur Stammler. Weber définit préliminairement la norme comme "l'affirmation générale d'un devoir-être (logique, éthique, esthétique)» (64). Cette première présentation montre que pour Weber une norme dans le sens le plus large est un contenu idéel "doté de valeur », que l'agir du sujet tend à réaliser parce qu'il le considère comme tel. Il est important de remarquer qu'ici Weber (qui pourtant dans la polémique avec Stammler s'intéresse surtout aux règles sanctionnées) lorsqu'il parle de "norme "se réfère en général à toute règle de comportement (sanctionnée ou non) dont le caractère impératif dérive directement du contenu sémantique de la règle, et non de son caractère instrumental pour la réalisation d'une fin donnée. Des exemples de Norm-Maximen dans ce sens large sont ainsi aussi bien des "normes de la pensée » (logique) et des «normes du beau » (esthétique), que des « normes du comportement correct» (éthique). Ce qui est commun à ces trois types de phénomènes est, premièrement, comme on a vu, qu'on a toujours affaire à un "devoir-être » (Sollen), une valeur digne de respect en soi et donc digne d'être poursuivie par le sujet, d'être réalisée : il s'agit d'impératifs, la norme s'impose pour elle-même et non en vue de quelque chose d'autre. Cet aspect est reflété par le sentiment d'obligation (Pflichtsgefühl) (65) que le sujet éprouve à l'égard de la norme. Deuxièmement, ces phénomènes ont en commun qu'ils sont tous des «idéaux », des «standards d'évaluation » (Wertungsstandards) $\mathrm{du}$ processus réel, toutefois leurs critères de jugement ne dérivent pas du rapport

(59) $W L$, pp. $323-324 ; 325 ; 329$.

(60) $W L$, p. 329.

(61) WL pp. 327-328; 334 ; 338 ; cf. aussi « Der Sinn der 'Wertfreiheit' der soziologischen und ökonomischen Wissenschaften » (1918), WL, pp. $517 ; 526 ; 529$.

(62) WL, p. 328.

(63) $W L$, p. 330.

(64) « Die generelle Aussage [...] eines (logischen, ethischen, ästhetischen) Sollens » : WL, p. 323.

(65) $W L$, pp. $334 ; 336$. 
entre moyens et fins, mais encore une fois du contenu même de la norme (66). Les critères ne sont donc pas «téléologiques », en vue de la fin de l'action, mais, selon la norme, logiques, ou esthétiques, ou encore éthiques (sittliche) (67).

Cette première définition wébérienne du concept de norme, construite à partir de la notion de «validité idéelle » (ideelle Geltung), est moins exclusive (donc moins forte théorétiquement) que la définition durkheimienne, mais elle comporte en même temps un élargissement de perspective important. Elle est moins exclusive, parce qu'elle ne se fonde pas sur le critère distinctif de la sanction, ce qui a le défaut de ne pas isoler conceptuellement les normes sociales des autres normes. Toutefois, cette "indétermination », qui met sous la même catégorie les normes sociales et les standards d'évaluation logiques, esthétiques, etc., a le mérite de porter au jour une lacune de la dichotomie durkheimienne : celle-ci partage le domaine des règles en "techniques" et "morales", mais, de cette manière, elle néglige deux faits fondamentaux : 1) il existe un grand nombre de «principes de conduite » qui sont bien des "règles", parce qu'elles engendrent des comportements dotés de sens, mais qui n'ont ni la forme « si tu veux $\mathrm{X}$, tu dois faire $\mathrm{Y}$ », ni ne sont garanties par des sanctions (par exemple, les règles de succession des plats dans un repas, ou les règles linguistiques, ou en général de formation d'expressions sensées : logiques, mathématiques, etc.). Du point de vue conceptuel, il faut donc faire une place à ce type de règles, et c'est ce que fait Weber, comme on verra. 2) L'idée de "norme », dans le langage commun, s'applique couramment aussi à des règles qui fondent des standards évaluatifs, mais qui ne sont pas garanties par des sanctions; la théorie doit en tenir compte, distinguant un premier concept, plus général, de norme, fondé sur la notion de "valeur idéelle », et un concept plus spécifique, que l'on peut référer aux normes sociales, fondé sur la notion de sanction.

Dans la théorie de Weber on trouve un cadre conceptuel plus complet, qui tient compte de ces dernières considérations, aussi bien dans les textes de sociologie juridique contenus dans Économie et société, que dans les «Concepts sociologiques fondamentaux »: la clé de voûte en est la typologie intérêt-coutumeconvention-droit, qui était bien claire à l'esprit de Weber déjà à l'époque de l'essai sur Stammler (1907), où elle est évoquée (68), pour ensuite être développée dans les textes écrits entre 1912 et 1914 (69), et reprise avec le même sens et la même terminologie en 1919-1920 pour les «Concepts sociologiques fondamentaux ». On peut donc donner une exposition unitaire de cette théorie.

Weber part du constat que dans la vie sociale on repère des régularités de l'agir ; ce qui intéresse les sociologues sont premièrement les régularités de l'agir doté de sens : dans la terminologie des "Concepts fondamentaux », on appelle « relation sociale » (soziale Beziehung) un agir social de plusieurs acteurs sociaux orientés par un sens subjectif (70). Ce sens peut être formulé par des « maximes »,

(66) WL, pp. $323 ; 329-330$.

(67) $W L$, p. 330.

(68) $W L$, pp. $343 ; 346 ; 352-353 ; 358$.

(69) On le verra dans l'analyse ; cf. aussi, pour la même période, « Über einige Kategorien der verstehenden Soziologie » (1913), WL, p. 445, n. 1.

(70) SG $(W u G)$, p. 13. 
qui sont les règles de conduite orientant le comportement des acteurs (71). À partir de cette base, on peut élucider une typologie de règles de conduite qui va d'une absence de sanction et d'une orientation vers le succès jusqu'à un maximum de sanction : les règles se rangent donc dans un spectre qui comprend $\mathrm{A}$ ) la « conduite par intérêt », B) la « coutume » (Sitte), C) la "convention" (Konvention), D) le « droit» (Recht) (72). Cette typologie, construite à partir des relations sociales, est beaucoup plus approchable de celle de Durkheim, qui elle aussi se sert de la sanction comme critère distinctif, et analyse les diverses conséquences de l'infraction à la règle pour en éclairer la nature. La position de Weber, toutefois, aboutit à une classification plus complexe parce que, tenant compte des problèmes que l'on vient de discuter, elle fait place aussi aux règles non instrumentales et non sanctionnées. Les quatre types que l'on vient d'énumérer sont d'abord différenciés en deux groupes de deux types chacun: l' «usage» (Brauch), A e B, et l'«ordre» (Ordnung), C e D. L'usage est une régularité de l'agir qui existe seulement en vertu d'une habitude de fait (73); l'ordre est en revanche une régularité qui existe en vertu de la représentation, de la part des acteurs, de la validité légitime de la règle de conduite : celle-ci est considérée comme un devoir-être et garantie par quelque forme de sanction (74). Les concepts de validité et de sanction, absents du premier groupe, servent de discrimination théorique.

Aux usages appartiennent A) la «conduite par intérêt» et B) la «coutume».

A) Dans la conduite par intérêt, la règle de conduite est donnée par des maximes instrumentales, qui indiquent les moyens à utiliser pour réaliser ses intérêts en tenant compte des intérêts des autres. En cas de violation de la règle, le résultat est l'insuccès et l'opposition éventuelle des intérêts des autres acteurs (75).

B) Dans la «coutume», les règles de conduite sont le produit d'habitudes acquises de longue date, que l'on suit le plus souvent de manière irréfléchie, par imitation, ou pour d'autres raisons plus ou moins conscientes, mais toujours « librement », c'est-à-dire sans être obligé par la pression sociale : la coutume n'est pas garantie extérieurement par des sanctions. En cas de violation de la règle, le comportement est «non adapté », l'acteur doit donc accepter des "incommodités » et des «adversités » plus ou moins grandes (76).

Aux ordres appartiennent C) les «conventions » et D) le «droit».

C) La convention est une règle de conduite considérée comme «valable » (dans le sens d'un idéal normatif) dans une communauté et garantie extérieurement par l'approbation ou désapprobation du milieu : elle est donc un principe digne de respect, un modèle de comportement, qui inspire (de façon variable) chez les acteurs un sentiment d'obligation. En cas d'infraction à la règle, le résultat est la désapprobation (Mißbilligung) du milieu, qui s'exprime de manières les plus diverses, mais, dans le cas de la convention, toujours sous un mode diffus et non

(71) SG $(W u G)$, p. 14.

(72) SG $(W u G)$, pp. 16-17.

(73) SG $(W u G)$, pp. 14-15.

(74) SG $(W u G)$, p. 16.

(75) $\mathrm{WgO}(W u G)$, pp. $190 ; 194$; SG $(W u G)$, p. 15.

(76) $\mathrm{WgO}(W u G)$, pp. $187 ; 190 ; 194$; SG $(W u G)$, pp. 15-16. 
organisé. La conséquence de cette désapprobation est la perte du respect de la part des autres et, souvent, aussi de soi-même (77).

D) Le droit a les mêmes caractères que la convention, sauf pour un point capital, à savoir que la sanction n'est pas la désapprobation diffuse et non organisée mais une punition définie et, surtout, administrée par un appareil coercitif stable (78).

\subsection{La validité sociale des normes : coutume, tradition, charisme, valeurs, intérêts}

Le concept wébérien de norme que l'on peut rapprocher de l'analyse durkheimienne est donc celui qui émerge de cette typologie : la norme sociale est une règle de conduite qui a le caractère du « devoir-être » et qui est garantie par des sanctions appliquées par la communauté contre celui qui l'enfreint. À partir de ce concept, il est possible d'aborder le point central de l'analyse wébérienne : le problème de la validité de fait des normes sociales, c'est-à-dire des facteurs qui stabilisent l'agir normatif. Cette question est, pour Weber, la question théorique fondamentale à ce sujet parce que sur une réponse correcte repose la possibilité d'une étude sociologique des conduites morales ; il y consacre ainsi de longs développements, souvent dans des contextes très polémiques. Weber vise à élaborer un cadre conceptuel « ouvert», qui rende possible de penser et d'articuler entre eux les différents facteurs qui peuvent stabiliser l'agir normatif, sans établir aucune priorité parmi eux ; la théorie ne fait qu'expliciter les types possibles et c'est une tâche de la recherche empirique, orientée par la théorie, de les repérer effectivement et d'en évaluer la portée causale, chaque fois différente (79).

Toutefois, la théorie wébérienne ne se borne pas à cette conception "formelle ": dans son analyse spécifique des facteurs qui stabilisent l'agir normatif, en effet, il est possible de percevoir aussi une théorie «substantielle», qui montre que le " poids » de tel ou tel facteur varie en fonction du processus de rationalisation. L'ensemble des déterminants possibles de l'agir normatif peut être donc disposé dans une échelle de "développement» selon laquelle certains facteurs, plus déterminants au début, s'affaiblissent ensuite, et d'autres ne deviennent possibles que grâce au processus de rationalisation. On verra qu'un moment originaire fondamental de cette échelle, constitutif pour tout type d'agir normatif, est celui de la tradition, dans lequel la force contraignante des normes est largement garantie « de l'intérieur» (des consciences), en vertu de la fusion entre ordres normatifs et sacré, caractéristique de ce type d'agir. On peut maintenant voir dans le détail comment se construit la théorie wébérienne.

Le point de départ est la conception de la validité empirique des normes comme représentation subjective. Comme Durkheim, Weber se meut dans un horizon de philosophie du sujet, c'est pourquoi il conçoit le «sens ", sur la base de la théorie rickertienne (80), en termes de rapports entre le sujet et l'objet: tout

(77) SS, pp. 443-444; WgO $(W u G)$, pp. 187-188; $191 ; 194$; SG $(W u G)$, pp. 17-18.

(78) $W L$ pp. $352-353$; WgO $(W u G)$, pp. $189-190$; SG $(W u G)$, pp. $18-19$.

(79) Cf. SG $(W u G)$, pp. 5-6; 9-10.

(80) Pour les rapports entre la sociologie de Weber et la philosophie de Rickert, cf. Peter-Ulrich Merz, Max Weber und Heinrich Rickert, Würzburg, Königshausen \& Neumann, 1990 ; en particulier, pour la question de la «validité » des jugements, pp. 128-129. 
contenu de sens est la « représentation », de la part du sujet, d'une validité. Pour ce qui concerne les normes, celles-ci sont des représentations référées à des valeurs. Or, Weber se sert de ces concepts pour fonder l'étude empirique des normes sociales: du point de vue des sciences sociales, celles-ci doivent être pensées comme des "représentations dans la tête " des acteurs sociaux, et lorsqu'elles sont efficaces dans la vie sociale ce n'est pas en vertu de leur valeur idéelle, ni parce qu'elles constitueraient le lien social, mais tout simplement parce que les acteurs, à un moment donné, dans une situation donnée, sont guidés par la représentation d'un devoir-être, considéré valable subjectivement, et tendent donc à conformer leur action au contenu sémantique de ce devoir-être (81). Cela permet de dire que l'efficacité réelle du contenu idéel de la norme n'est due qu'à la conviction subjective de sa validité de la part des acteurs. En outre, la référence au " sens subjectif », " intentionné », de la norme impose au chercheur de tenir compte de deux autres circonstances : 1) le sens intentionné par un acteur peut ne pas coïncider exactement avec celui des autres acteurs, il peut varier selon l'interprétation et la position sociale, ce qui veut dire que, lorsqu'il y a efficacité empirique du contenu idéel de la norme, celui-ci (dans son incarnation effective) peut être très éloigné de la représentation qu'en donnerait un point de vue «dogmatique » (éthique ou juridique) ; 2) si la norme est la représentation d'un sujet, cela veut dire que celui-ci peut avoir plusieurs raisons pour accepter cette représentation et s'y conformer; c'est dire qu'on ne peut pas conclure de cette conformité du comportement que la norme est considérée « juste » par le sujet (82).

Ce cadre conceptuel de base permet aisément d'affirmer le principe non seulement que la norme n'est qu'une composante possible de l'agir réel en général, mais aussi que, même dans le cas spécifique de l'agir normatif, le contenu idéel de la norme n'est qu'une des causes possibles de cet agir (83). Quelles sont ces motivations possibles de l'agir normatif? Weber souligne avec insistance, en plusieurs endroits de son œuvre, qu'il y a souvent de nombreuses motivations même pour un seul acte normatif, et chaque fois il en donne une liste, plus ou moins longue. Pour synthétiser ces différentes énumérations, il est utile dans un premier temps de séparer les textes considérés ici en deux groupes chronologiques: le premier comprend ceux composés entre 1907 et 1914, avant la dernière révision d'Économie et société, et le deuxième comprend le résultat de cette révision conceptuelle, c'est-à-dire les sections initiales de cette œuvre, écrites en 19191920. Si on synthétise les énumérations du premier groupe, on arrive à la liste suivante : on peut se conformer à une norme, dit Weber, a) inconsciemment, par « habitude »; b) par respect de la «tradition»; c) par adhésion à un principe " religieux » ou « éthique "; d) par crainte de la " désapprobation » ou " sanction » de la communauté ; e) en vue d'un "intérêt» (84). Ces motivations possibles sont normalement présentées de manière non systématique, sans établir un ordre entre elles; toutefois, anticipons que l'ordre de succession proposé ici n'est pas dû au hasard, mais respecte une succession élaborée par Weber lui-même, dans d'autres

(81) Cf. $W L$, pp. 334-335; 346-348; SG $(W u G)$, p. 16.

(82) $W L$, pp. 334-335; 347-348.

(83) $W L$, p. 330 ; SS, pp. $476 s q$; WgO $(W u G)$, p. 181 ; SG $(W u G)$, pp. 5-6.

(84) WL, pp. 330-331; WgO $(W u G)$, pp. $182 ; 183-184 ; 189 ; 194$. 
endroits de son œuvre, et que l'on justifiera en exposant l'analyse " généalogique » des fondements de validité des normes (85). Dans les textes de la deuxième période, les motivations possibles de l'agir normatif sont présentées d'abord du point de vue de leur efficacité dans la stabilisation des ordres légitimes (legitimen Ordnungen) : ceux-ci, en effet, peuvent être suivis ou bien 1) par croyance en leur « légitimité » (Legitimität), ou bien 2) par la force de l'habitude, de la coutume (Sitte), ou bien 3) pour des motivations rationnelles par rapport aux fins (zweckrational). L'échelle entre les trois possibilités est ici explicitée, car Weber souligne que les ordres les plus stables sont ceux qui peuvent compter sur 1), en plus de 2) et 3), et que ceux qui se fondent au moins aussi sur 2) sont plus stables que ceux fondés seulement sur 3) (ces derniers sont définis «weit labiler» par rapport à 2) et «noch ungleich labiler» par rapport à 1)) (86). Comme on sait, en outre, que la légitimité d'un ordre normatif peut être fondée sur la «tradition», la «foi» (affective-religieuse ou éthique) et la «légalité » (87), on peut compléter la liste qu'on vient de citer, en suivant toujours le sens du plus stable au moins stable : 1a) légitimité par tradition; 1b) légitimité par foi (affective-religieuse ou éthique); 1c) légitimité par légalité ; 2) la coutume; 3) les motivations rationnelles par rapport aux fins. Dans quel rapport se trouve cette présentation avec une autre, qui devrait avoir la même signification, mais qui en est cependant un peu différente ? Weber affirme en effet ailleurs que la légitimité d'un ordre peut être garantie (c'est-à-dire, stabilisée) ou bien de l'intérieur (innerlich), et dans ce cas a) affectivement, b) rationnellement par rapport aux valeurs, c) religieusement, ou bien de l'extérieur (äußerlich), et dans ce cas d) par désapprobation et coercition, e) par intérêt (88). On voit bien ici que l'échelle va de l'adhésion la plus intérieure (l'identification affective) à l'acceptation la plus extérieure (le calcul des intérêts) et que cette progression est différente de celle qui va du plus stable au moins stable. On est frappé par l'absence de la «coutume» et de la «tradition» (que, à ce point, je n'arrive pas à expliquer) et par la position du « religieux », qui est placé après le wertrational, à la fin des motivations internes, plus près de celles externes (89). Cette position du religieux s'explique toutefois parce que, ici, les motivations religieuses d'adhésion à la norme ne correspondent pas à la « foi dans une révélation » (qui relève de l'agir affectif, c'est-à-dire de la légitimation par charisme), mais dérivent de la conviction que, pour obtenir les «biens du salut» (Heilsgüter), il faut respecter certaines normes : la conduite normative est ici assujettie à la réalisation du salut, sur la base d'un raisonnement qui s'approche de la rationalité par rapport aux fins. En outre, Weber remarque que précisément par le monopole des biens du salut la hiérocratie exerce une "coercition psychique » (pshychische Zwang) sur les fidèles ; c'est pourquoi ici la motivation religieuse, tout en étant encore intérieure, se trouve à la frontière des motivations extérieures (90). Compte tenu de ces considérations, on remarque que cette deuxième série de la période 1919-1920 se rapproche peut-être plus de celle de 1907-1914, qui peut donc être lue à la lumière du principe interne-externe.

(85) Cf. infra, 2.3.

(86) SG $(W u G)$, p. 16 ; TdH $(W u G)$, p. 122.

(87) SG $(W u G)$, pp. 19-20.

(88) SG $(W u G)$, pp. 17-18.

(89) Cf. les remarques de WeBer: SG $(W u G)$, p. 19.

(90) SG $(W u G)$, p. 29. 
Que peut-on conclure en général de ces analyses ? 1) D’abord, les «motivations possibles » de l'adhésion à un ordre légitime, si on synthétise tous les textes sans expliciter le principe de la série, sont les suivantes : l'habitude irréfléchie, la pure coutume ; la tradition ; la foi (affective) en une révélation ; la foi (rationnelle par rapport aux valeurs) en un principe éthique; la poursuite des biens du salut; la crainte de la sanction sociale ; les intérêts individuels. 2) Deuxièmement, il y a une différence marquante entre une présentation de ces facteurs du point de vue de la " stabilité » des ordres (91), et une présentation du point de vue du caractère plus ou moins interne de l'adhésion (92), car la coutume (Sitte) et la tradition, qui dans le premier cas se trouvent vers la fin, disparaissent dans le deuxième. 3) Troisièmement, la série de 1912-1914 ressemble toutefois beaucoup plus à la présentation selon le critère interne-externe, mais, si on les unit (comme on a fait au début de cet alinéa) on voit que coutume et tradition se trouvent au début. Pour donner une interprétation générale de ces présentations et les synthétiser, je propose l'hypothèse suivante : dès 1912, et avec constance jusqu'à 1920, lorsqu'il formule ces problèmes, Weber présuppose une succession de principe générale, qui est la perspective la plus importante, dans sa sociologie, pour expliquer la validité de fait des normes sociales et, surtout, sa genèse: cette succession est celle qu'on peut orienter $d u$ "moins réflexif " au "plus réflexif ", qui correspond à une tendance fondamentale du processus de rationalisation et qui explique la position différente de la Sitte dans les deux séries. On propose donc comme perspective privilégiée celle qui va du pôle de la Sitte à celui de l'agir zweckrational. Comment peut-on justifier cette thèse interprétative ? Les bases textuelles qu'on peut porter à l'appui sont les suivantes. Dans l'étude sur Stammler, ainsi que dans les textes d'Économie et société composés entre 1912 et 1914 , Weber développe à plusieurs reprises une thèse « évolutionniste» selon laquelle à l'origine du comportement normatif se trouvent des régularités (Regelmäßigkeiten) de l'agir produites par «adaptation» au milieu; la peur de «dévier» par rapport à ces habitudes les transformerait ensuite en "préceptes", renforcés par la peur des sanctions collectives et divines (93). On analysera mieux ces thèses dans la prochaine section de cet article, pour montrer de quelle façon Weber conçoit la «sacralité de la tradition ». Il est important toutefois de citer immédiatement un passage de ces textes, pour voir le rôle « originaire" que Weber attribue à l'habitude: "Plus on remonte dans le temps, plus l'allure du comportement [...] est déterminée exclusivement par rapport aux 'habitudes existantes', simplement comme telles [...]» (94). Le pur habituel, et donc la Sitte, se trouve ainsi à l'origine de toute uniformité de l'agir, et en particulier - comme on verra - de l'agir normatif. Ce cadre interprétatif général reste valable aussi pour les textes écrits en 1919-1920, où l'on trouve même des remarques qui éclairent la signification conceptuelle du processus, et sa portée pour interpréter les différentes motivations possibles de l'agir normatif. D'abord, dans la célèbre typologie de l'agir social (traditional, affektuell, wertrational, zweckrational)

(91) SG $(W u G)$, p. 16 ; TdH $(W u G)$, pp. 122-124.

(92) SG $(W u G)$, pp. 17-19.

(93) $W L$ p. $378 ; \mathrm{WgO}(W u G)$, pp. $188 ; 191-192 ; 195-196$.

(94) «Je weiter rückwärts, desto umfassender ist die Art des Handelns [...] ausschließlich durch die Eingestelltheit auf das 'Gewohnte' rein als solches bestimmt »: $\mathrm{WgO}(W u G)$, p. 188 (souligné par moi). 
on sait que, bizarrement, Weber considère l'agir traditionnel comme « à la limite » de l'agir doté de sens; si on tient compte du schéma évolutif dont on parle, cette remarque devient compréhensible : l'agir traditionnel est presque au niveau de la simple habitude, il est presque totalement irréfléchi, et son extrême opposé est, généalogiquement et logiquement, l'agir rationnel par rapport aux fins (95). Cette opposition «polaire» est explicitée peu après, dans l'analyse de la Sitte, et liée immédiatement à la thèse " évolutive » : " [Le fait de se guider sur les seuls intérêts] constitue, par la prise de conscience et le laisser-aller interne qui le caractérisent, l'exacte antithèse à toute espèce de contrainte interne par insertion dans une simple coutume, familière, [...]. Un des éléments essentiels de la rationalisation de l'agir consiste précisément dans la substitution d'une adaptation méthodique commandée par les situations d'intérêt à l'insertion intime dans une coutume familière » (96). Donc : la coutume et l'agir rationnel par rapport aux fins sont les types polairement opposés de l'agir, et se placent dans une direction de rationalisation qui va de la première au dernier (en passant par l'agir affectif et par l'agir rationnel par rapport aux valeurs (97)). Tout cela éclaire bien quel est le critère général qui oriente cette succession, ainsi que l'ordre de la typologie générale de l'agir : on va dans le sens d'une adaptation de plus en plus consciente aux conditions externes. Il y a donc, dans la succession, une augmentation d' êtreconscient »(Bewusstheit) et de réflexivité : les déterminantes normatives de l'agir perdent leur condition originaire de régularités irréfléchies, pour devenir progressivement des "représentations conscientes du devoir-être » (rationalité par rapport aux valeurs) et, enfin, des "représentations disponibles », " objectivables » en fonction des fins de l'action. Il y a accroissement de réflexivité et d'objectivation à la fois. Ces considérations plus générales sont confirmées par d'autres remarques éparses : toujours dans les «Concepts fondamentaux» on définit la coutume «le type le plus fréquent de disposition intérieure » (98), et dans les typologies du pouvoir on reprend l'opposition «évolutive» qui va « de l'habitude inconsciente [...] jusqu'aux considérations purement rationnelles par rapport aux fins » (99).

Sur cette base textuelle, on peut donc affirmer que la perspective privilégiée pour interpréter les motivations possibles de l'agir normatif est celle qui les dispose dans une série qui va du moins au plus réflexif. Dans cette perspective, la coutume se trouve au début; le fait que, si on adopte au contraire la perspective du plus au moins stable, la coutume se trouve vers la fin (juste avant l'intérêt), montre un

(95) $\mathrm{SG}(W u G)$, p. 12

(96) « [Die Orientierung an Interessenlagen] bildet in ihrer Bewußtheit und inneren Ungebundenheit den polaren Gegensatz gegen jede Art von innerer Bindung durch Einfügung in bloße eingelebte 'Sitte' [...]. Eine wesentliche Komponente der 'Rationalisierung' des Handelns ist der Ersatz der inneren Einfügung in eingelebte Sitte durch die planmäßige Anpassung an Interessenlagen » : SG $(W u G)$, p. 15.

(97) Cf. la phrase suivante, SG $(W u G)$, pp. 15-16: « [La rationalisation de l'agir] peut se développer [...] négativement au détriment de l'agir affectif si ce n'est à celui de la coutume ou encore au profit d'un agir purement rationnel par rapport aux fins, qui ne croit pas aux valeurs, et au détriment d'un agir lié à la rationalité par rapport aux valeurs » (" [die 'Rationalisierung' des Handelns kann] außer auf Kosten der Sitte auch auf Kosten affektuellen Handelns, und endlich auch zugunsten eines wertungläubigen, rein zweckrationalen, auf Kosten wertrational gebundenen Handelns verlaufen »; souligné par moi).

(98) « Die von allen häufigste Art der inneren Haltung »: SG $(W u G)$, p. 16. p. 122

(99) «Von dumpfer Gewöhnung [...] bis zu rein zweckrationalen Erwägungen »: TdH $(W u G)$, 
résultat important de cette conception "évolutive » : les régularités initiales, bien que fortes, deviennent réellement impératives et touchent immédiatement le point le plus élevé de force contraignante normative dès qu'elles sont investies par la magie et par la religion, et se transforment ainsi, d' " habitudes", en "tradition (sacrée) ». Celle-ci a une toute autre signification que les simples habitudes, et se trouve toujours au début de la série, car elle correspond au maximum de stabilité avec le minimum d'《 objectivabilité » des normes.

\subsection{La genèse du comportement religieux : le sacré}

On peut maintenant aborder le dernier point de cette analyse : la genèse des liens normatifs selon Weber, qui constitue le soubassement de sa vision de leur validité factuelle. On approfondira les deux premiers "moments » dans la typologie générale des motivations possibles d'acceptation d'une norme, qui représentent les premières étapes d'un processus de développement: (a) l'habitude, ou coutume, et (b) la tradition.

(a) On a déjà vu que, selon Weber, plus on remonte en arrière dans l'histoire de l'humanité, plus on trouve comme forme de comportement dominant l'habitude, la répétition irréfléchie de comportements acquis (100). Ces premières formes de régularité du comportement social ont, selon Weber, plusieurs origines, qui expliquent leur force et leur stabilité : 1) des actions conditionnées organiquement (101); 2) des actions adaptées de manière irréfléchie aux conditions extérieures de vie, à travers des processus de réactions à des stimulations (102) ; 3 ) des processus irréfléchis d'imitation du comportement des autres, des « comportements de masse »(103). Ces « régularités de fait», qui ne sont pas encore orientées par un sens, exercent toutefois une contrainte sur les individus, qui se manifeste par la peur (presque instinctuelle, vague, indéterminée) de changer le " cours des choses ». L'origine de cette peur est une sorte de "disposition» (Eingestelltheit) à préserver un comportement acquis : la déviation de cette couche pré-symbolique du comportement est donc perçue comme quelque chose d' " inquiétant» (beunruhigend) et a des effets psychiques qui ressemblent à des «troubles de fonctions organiques" (Störungen organischer Funktionen) (104). C'est de cette première forme de " contrainte psychologique » que, selon Weber, naît progressivement, à travers un processus qu'il n'analyse pas, la «représentation » du caractère normatif de ces régularités, de leur caractère " obligatoire » (Verbindlichkeit) (105). On a ainsi la première forme - la plus indéterminée, encore - du passage de la «coutume» à la «tradition». Deux autres causes peuvent concourir à ce passage, causes qui sont souvent mêlées entre elles : la crainte de susciter l'opposition d'autres hommes qui suivent ce comportement, donc la crainte de la réaction du milieu ; cette crainte se transforme parfois en peur

(100) $\mathrm{WgO}(W u G)$, p. 188.

(101) $\mathrm{WgO}(W u G)$, p. 192.

(102) $\mathrm{WgO}(W u G)$, pp. $192 ; 195-196$.

(103) $\mathrm{WgO}(W u G)$, p. 192 ; SG $(W u G)$, pp. 11-12.

(104) $\mathrm{WgO}(W u G)$, p. 188.

(105) WgO $(W u G)$, p. 192. 
de susciter une réaction des «puissances suprasensibles » (106). Cette thèse ressemble curieusement à la thèse durkheimienne sur l'origine sociale du sacrénormatif; mais Weber n'y revient pas particulièrement. À vrai dire, un problème théorique se pose ici. On a vu que Weber propose une thèse, pour ainsi dire, « comportementaliste » à l'origine de l'agir normatif; en même temps, il semble dire qu'il ne s'agit pas de la seule origine, qu'il faut également attribuer un rôle à la société et à la religion. Toutefois, les relations entre ces trois éléments ne sont pas tout à fait claires, et surtout ne sont pas clairs les rapports entre les thèses exposées jusqu'ici (contenues dans les sections «juridiques » d'Économie et société) et les thèses sur le lien originel entre la religion (le sacré) et les normes (développées dans les écrits de Sociologie de la religion, aussi bien dans la section correspondante d'Économie et société, que dans le Recueil d'études de sociologie de la religion). Dans le premier cas, en effet, Weber semble privilégier le primat de l' "adaptation », et parle du religieux comme d'une sorte de "projection » des phases originaires de constitution de l'agir humain; dans les autres textes, en revanche, il semble plus prudent, et montre de manière approfondie le développement « immanent» du phénomène religieux.

La «Sociologie de la religion» d'Économie et société, confirmant ce qu'on vient de dire, ouvre l'étude sur l'origine des religions avec une approche ambiguë ; d'un côté, on a l'impression de pouvoir rattacher immédiatement cette analyse aux thèses «naturalistes » qu'on vient de citer, car Weber affirme que, à l'origine, l'agir magique et religieux avait des fins «terrestres », qu'il était produit par la recherche du bien-être dans l'ici-bas (Diesseits); d'un autre côté, le sens même de cette affirmation est en contradiction avec ce naturalisme, parce qu'elle est obtenue à partir du principe méthodologique, énoncé préliminairement, qu'il faut étudier l'agir religieux sur la base du "sens subjectif ». Conséquemment à cette position, Weber montre tout de suite que l'orientation au bien-être d'ici bas, propre au comportement magique, n'est tel que grâce à la «vision du monde » magique, fondée sur le présupposé de la séparation du monde en forces exceptionnelles (magiques) et forces ordinaires (107). Cette deuxième partie du raisonnement équivaut à l'inversion de la première : c'est parce qu'on pense le monde selon les catégories magiques que certaines pratiques sont « utiles» pour l'ici-bas. Weber le reconnaît lui-même, en observant que ces pratiques présupposent déjà une abstraction (un germe de dualisme), pour faible qu'elle soit (108), et que d'ici naît l'idée des «forces extraordinaires ». L'ambiguiité entre les deux attitudes méthodologiques - l'analyse immanente des structures de sens et de leurs transformations, la tentative de les ramener à des causes externes - est présente dans tout le texte ; il ne s'agit pas ici de juger si elle est bien ou mal résolue "en général », mais seulement de montrer qu'elle ne l'est pas pour l'origine du religieux et de l'agir normatif. C'est pourquoi on se limitera à montrer quelques points intéressants de l'analyse « immanente ».

La première phase du comportement religieux est donc la «magie ». Il n'est pas inexact de dire qu'ici, et avec l'introduction du concept de charisme, Weber

(106) $W L$, p. 378 ; WgO $(W u G)$, p. 189.

(107) RelS $(W u G)$, pp. 245-246.

(108) RelS $(W u G)$, p. 246. 
cherche à formuler la nature du «sacré » et de ses rapports avec le "profane ». L'intérêt par rapport à Durkheim, c'est que Weber différencie le concept de sacré en fonction du développement historique des religions. La première définition qu'il donne lie le sacré à l' «extra-quotidien» et aux «forces extraordinaires ». Pour l'agir magique, dit Weber, le partage fondamental est celui entre " quotidien » et « extra-quotidien » : ce dernier est tout ce qui est capable de produire des phénomènes exceptionnels désirés (l'extase, des effets météorologiques, thérapeutiques, divinatoires, etc.). Il s'agit donc d'un "don » (Gabe), d'une qualité intrinsèque à certains objets et à certaines personnes, et que Weber appelle "charisme ». C'est le sacré en tant que force impersonnelle qui «habite» ces objets et ces personnes (109). La présence de cette force est déjà une première abstraction, c'est pourquoi elle est pensée sous forme d'«esprits»; toutefois, ces esprits agissent comme une force naturelle, bien qu'exceptionnelle : ils sont « matériels », bien que « invisibles »; " impersonnels », bien que dotés d'une sorte de volonté. C'est pour ces raisons que l'agir magique a la structure, dans un certain sens, de l'agir rationnel par rapport aux fins : il n'est pas en effet un agir "éthique », mais plutôt une technique de "manipulation » directe de ces forces.

C'est ici qu'on trouve la première origine religieuse de l'agir normatif, selon Weber : les pratiques magiques sont des techniques pour influencer positivement les esprits, des techniques qui ont réussi, et qui donc sont « non modifiables », sous peine de déchaîner des réactions de la part des esprits (110). Ces pratiques deviennent ainsi le noyau d'un comportement «sacré », dont le caractère contraignant est garanti par la crainte de la réaction des «forces surnaturelles ». En ces termes, la thèse se rattache directement aux thèses «naturalistes » exposées dans les autres sections d'Économie et société; il faut toutefois rappeler qu'en réalité elle dépend aussi d'une fondation originellement religieuse, dont la racine n'est pas éclairée par Weber, c'est-à-dire la constitution magique du monde, qui distingue les forces « naturelles » et les forces « extraordinaires ».

(b) Le passage au comportement normatif stricto sensu n'est toutefois pas encore accompli ; ce comportement, comme on a vu, se différencie catégorialement de tout agir « instrumental », car celui-ci est fondé sur des Zweck-Maximen (ce qui est le cas aussi de l'agir magique), alors que le premier est fondé sur des NormMaximen. Pour que naisse effectivement l'agir normatif, il faut que s'affirme cette différenciation entre règles techniques et règles pratiques. Elle est encore absente dans la magie, en raison de la conception des esprits comme des forces que l'on peut influencer ; elle n'apparaît qu'au moment où le processus d'abstraction, déjà entamé avec l'idée d'esprits, évolue vers une conception de l' «âme», des « dieux » et des «démons ». Ceux-ci ne sont plus des forces «dans» les choses, mais des «puissances surnaturelles» (übernatürliche Mächte) (111) séparées du monde. Dans le passage du «naturalisme pré-animiste» à ce nouveau stade

(109) Ici Weber se réfère directement, comme Durkheim, au mana: RelS (WuG), p. 245 ; Durkheim et Weber acceptent la thèse, largement diffuse à leur époque, de l'universalité du mana: pour une critique de cette théorie, aujourd'hui discréditée, cf. déjà Mircea Eliade, Traité d'histoire des religions, Paris, Payot, 1949. Pour l'interprétation du charisme, dans ce texte, comme " sacré », cf. Wolfgang Schluchter, Religion und Lebensführung. II, Frankfurt a. M., Suhrkamp, 1988, pp. 142-143.

(110) RelS (WuG), pp. 257-258.

(111) RelS ( $W u G)$, p. 247. 
s'accomplit le dualisme déjà implicite dans le premier, mais pas encore développé dans ses conséquences : la sphère du religieux se fonde maintenant sur un "monde divin » purement spirituel, au-delà du monde sensible, qui lui est assujetti. Les forces divines n'ont plus l'essence - manipulable - des forces naturelles, mais une volonté et une signification; lorsqu'elles se manifestent dans le monde d'ici-bas, elles ne sont plus "dans» les objets, mais elles les «commandent».

Le comportement religieux dans son sens spécifique, comme agir guidé par la représentation des biens du salut, et donc de nature "éthique », naît seulement à ce stade : «Nous enregistrons simplement ici le fait que le résultat de ce processus est l'origine, d'une part, de l' âme', d'autre part, des 'dieux' et des 'démons', donc des puissances 'surnaturelles'. Régler leurs rapports avec les hommes constitue le domaine de l'agir 'religieux'»(112). Ce passage est un vrai "saut qualitatif», parce qu'il introduit la différenciation entre agir magique et agir rituel-symbolique, qui conduit à son tour à la différenciation, constitutive de tout agir normatif, entre règles techniques et règles pratiques. L'origine de ces différenciations est le dualisme entre le monde divin et le monde terrestre; le monde ne se réduit plus à ce qu'on peut percevoir avec les sens : «Si derrière les événements et les choses réels se cache quelque chose d'autre, de particulier, de spirituel dont ils ne sont que le symptôme, ou même le symbole, ce n'est pas sur ceux-ci qu'il faut tenter d'influer. Ce sont les puissances qui s'extériorisent par eux qu'il faut influencer à l'aide de moyens qui puissent parler à un esprit ou à une âme, donc de moyens qui 'signifient' quelque chose : les symboles »(113). L'agir religieux abandonne progressivement (ou laisse survivre en position subordonnée) les pratiques magiques, pour assumer des pratiques symboliques, parce que le rapport avec le « divin» n'est plus celui qui cherche à " influencer » la divinité, mais celui de l' « assujettissement à son vouloir» : on passe de la «coercition du dieu» (Gotteszwang) au « service du dieu » (Gottesdienst) (114). Les actions « sacrées », qui garantissent le bon vouloir des dieux, sont maintenant des rituels symboliques, qui remplacent les choses sacrées par des simulacres ou par des rites "symbolisant » l'au-delà ; ces actions-là deviennent alors «intouchables », " non modifiables », et puisqu'elles sont de nature symbolique, elles se transforment en "impératifs", en "prescriptions de conduite » rendues contraignantes par la terreur du châtiment divin et par le désir de s'approcher du principe divin. C'est de cette manière qu'on trouve, chez Weber comme chez Durkheim, une théorie de l'origine sacrale du comportement normatif : ce processus, en effet, amène à la naissance de la tradition, c'est-à-dire d'une régularité de l'agir produite par la représentation de la légitimité de ce qui vient du passé. Or, la tradition est un "saut qualitatif» par rapport à la simple coutume, parce que la force des normes traditionnelles dérive de leur caractère sacré. Le passé est conçu comme «sacré » et digne de respect en soi (115), parce

(112) «Wir registrieren hier lediglich als Resultat des Prozesses die Entstehung einerseits der 'Seele', andererseits der 'Götter' und 'Dämonen', 'übernatürlicher' Mächte also, deren Beziehungen zu den Menschen zu ordnen nun das Reich der 'religiösen’ Handelns ausmacht » : RelS ( WuG), p. 247 (souligné par Weber); cf. Aussi Wolfgang Schluchter, Religion und Lebensführung. II, op. cit., p. 25.

(113) «Steckt hinter den realen Dingen und Vorgängen noch etwas anderes, Eigentliches, Seelenhaftes, dessen Symptome oder gar nur Symbole jene sind, so muß man nicht die Symptome oder Symbole, sondern die Macht, die sich in ihnen äußert, zu beeinflussen suchen durch Mittel, die zu einem Geist oder einer Seele sprechen, also etwas 'bedeuten' : durch Symbole »: RelS $(W u G)$, p. 248.

(114) RelS $(W u G)$ pp. 257-258.

(115) SG $(W u G)$, pp. $12 ; 19$. 
qu'il est le produit des pratiques rituelles symboliques qui assurent l'harmonie entre le monde divin et le monde terrestre, et qui ne peuvent être modifiées, même dans les plus petits détails, sans provoquer l'ire des dieux et la perte du salut: « L'effet premier et fondamental des représentations 'religieuses' sur la conduite de la vie et sur l'agir économique a donc été, en général, stéréotypisant. Toute modification d'un usage qui s'accomplit, d'une façon ou d'une autre, sous la protection des forces surnaturelles peut affecter les intérêts des esprits ou des dieux. Aux incertitudes et aux résistances que rencontre tout novateur, la religion ajoute des obstacles puissants. Le sacré est, spécifiquement, l'immuable» (116).

\section{Conclusion : de Weber à Durkheim}

Ces analyses des théories normatives durkheimienne et wébérienne sont encore très incomplètes, elles ne font qu'en ébaucher les premiers fondements. C'est pourquoi on se limitera dans cette conclusion à quelques remarques générales, pour montrer quelles sont les directions théoriques fécondes. On prendra comme fil conducteur la position de Weber, dont on confrontera les résultats aux conclusions partielles sur Durkheim (117).

On peut distinguer, chez Weber comme chez Durkheim, le niveau des concepts fondamentaux de celui de la théorie explicative. Pour le premier, on trouve un concept de norme très articulé, plus riche et « ouvert » que celui de Durkheim. Les « gains théoriques » de cette approche sont deux : 1) le concept wébérien de norme fait une place conceptuelle à l'agir régulé non sanctionné, mais non instrumental (la «coutume» et les «normes» de la pensée, esthétiques, etc.) ; 2) il permet de mieux penser la "composition » de plusieurs motivations, y compris non normatives, pour expliquer la stabilité de l'agir normatif. Toutefois, on perd ici, par rapport à la conception durkheimienne, l'outil conceptuel de la «désirabilité » (118); certes, celle-ci n'est pas tout à fait absente, puisque Weber parle d' " exemplarité » (Vorbildlichkeit) des normes (119), mais elle n'est pas développée théoriquement. C'est pourquoi il ne semble pas percevoir l'importance de l'expérience de «transcendance de soi » dans la norme, ni le rôle qu'elle joue dans la constitution de l'identité. Il est vrai que l'intégration de cette perspective dans la conception wébérienne ne va pas de soi : la pluralité de motivations possibles, comme source de stabilisation de l'agir normatif, présuppose dans l'acteur un

(116) «Die erste und grundlegende Einwirkung 'religioser' Vorstellungskreise auf die Lebensführung und die Wirtschaft ist also generell stereotypierend. Jede Aenderung eines Brauches, der irgendwie unter dem Schutz übersinnlicher Mächte sich vollzieht, kann die Interessen von Geistern und Göttern berühren. Zu den natürlichen Unsicherheiten und Gehemmtheiten jedes Neuerers fügt so die Religion mächtige Hemmungen hinzu : das Heilige ist das spezifisch Unveränderliche » : RelS (WuG), p. 249 (souligné par moi); cf. aussi RechtS (WuG), pp. 446 ; 459 sq. ; 472 sq., et RS I, pp. 269-271.

(117) Cf. supra, 1.3.

(118) Pour une reprise contemporaine de ce concept, cf. Heinrich PoPITZ, Die normative Konstruktion von Gesellschaft, Tübingen, Mohr, 1980, pp. 10-11.

(119) SG $(W u G)$, p. 16. 
certain détachement à l'égard de la norme, qui devient ainsi pour lui plus ou moins « disponible ». Le cadre conceptuel général donne l'impression qu'il faut toujours présupposer cette possibilité (du détachement "pragmatique ») dans le comportement de l'acteur; cette perspective est inconciliable avec un rôle constitutif de la norme à l'égard de l'identité de l'acteur. On retombe dans le dilemme théorique déjà évoqué : comment penser l'action des facteurs causaux «extérieurs » à la norme si celle-ci est constitutive ?

Le second niveau, l'explicatif, offre chez Weber une première réponse à cette question. On a vu en effet que l'explication de la validité factuelle des normes est pour lui indissociable du processus de rationalisation. Le cadre conceptuel général, fondé sur la possibilité du détachement, est en réalité un résultat de ce processus ; on a vu que l'importance des facteurs irréfléchis et, surtout, magico-religieux est beaucoup plus forte au début de ce processus, quand ces facteurs sont « constitutifs » de l'agir normatif. C'est pourquoi on peut affirmer qu'on trouve chez Weber une théorie de la «fondation sacrale » des normes. Toutefois, cette thèse est assez différente de la thèse durkheimienne, car elle n'est pas construite à partir d'une généralisation du couple sacré/profane, mais à travers une analyse "évolutive », qui montre la naissance de la «tradition sacrée» par la sortie du monde magique. La différenciation historique du sacré est un point central de la sociologie wébérienne : cela lui permet d'étudier les rapports entre les conceptions du divin et les conduites de vie, et d'expliquer ainsi la formation d'un univers éthique de plus en plus autonome (120). Elle permet en outre d'éviter les pièges d'une construction unitaire du concept de sacré, qui tend à en reproduire la conception chrétienne (121).

On trouve également chez Weber une théorie sociologique sur l'origine du religieux, une tentative de ramener la crainte des puissances surnaturelles (et donc la source du normatif) à une «disposition » naturelle de conservation des habitudes acquises, et à la crainte de la pression sociale. Toutefois, l'approche wébérienne montre ici beaucoup plus que celle de Durkheim les limites de la philosophie du sujet. Dans sa rigoureuse cohérence logique, Weber refuse toute représentation mentale qui ne soit pas le produit du rapport entre le sujet et l'objet, et cherche la source du normatif dans un mécanisme d'adaptation individuelle au milieu. De cette manière, il perd de vue ce qui était au contraire bien clair pour Durkheim : le caractère trans-subjectif des normes, leur transcendance par rapport au sujet, qui exprime une dimension différente aussi bien des "états des choses" que des « représentations subjectives » (cela est valable non seulement pour les normes, mais pour le langage, la logique, les connaissances, etc. (122)). C'est la dimension des significations, qui s'explique de façon raisonnable en prenant comme base

(120) Cf. Wolfgang Schluchter, Religion und Lebensführung. I, op. cit., pp. 219-273.

(121) Cf. Danièle Hervieu-LÉGer, La religion pour mémoire, op. cit. ; Carsten ColPe, Über das Heilige, op. cit.

(122) Cf., à ce sujet, la grande difficulté de Weber à penser sociologiquement le langage : « Über einige Kategorien der verstehenden Soziologie » (1913), WL, pp. 452 sq. Sur l'autonomie de la dimension des significations, cf. Karl R. PopPER, " On the Theory of the Objective Mind », (1968), in Karl R. Popper, Objective Knowledge, Oxford, Clarendon Press, 1972, pp. 153-190, et la reprise critique de Jürgen Habermas, Theorie des kommunikativen Handelns. I, Frankfurt a. M., Suhrkamp, 1981, pp. 115126. 
théorique l'apprentissage social des règles, et non le rapport solipsiste du sujet à l'objet (123). Chez Durkheim aussi, on l'a bien vu, la reconnaissance ultime de cette dimension manque parce que l'origine de la socialisation se trouve dans des mécanismes - les "effervescences collectives »-qui ont justement la fonction d'expliquer la sortie du sujet de soi-même. Mais avant d'atteindre ce dernier niveau explicatif, toute sa sociologie est, comme on sait bien, un commentaire de l'existence d'un monde " tiers », entre celui du sujet et celui de l'objet. La reprise de sa théorie normative peut aider à dépasser le rigide dualisme wébérien, à condition toutefois de repenser radicalement les concepts de règle et de signification.

Mauro PIRAS

Turin

(123) Sur la constitution sociale des règles, cf. Ludwig Wittgenstein, Philosophische Untersuchungen, in Ludwig Wittgenstein, Werkausgabe. I, Frankfurt a. M., Suhrkamp, 1984, pp. 225-618 : $\S \S 82-86,143-154,185-189,198-202$, 206-212, 238. 
Résumé

L'article cherche à poser les premiers jalons d'une théorie de l'efficacité sociale des normes, en s'appuyant sur la conception durkheimienne du sacré. On reconstruit ainsi le concept de norme chez Durkheim et Weber, et l'explication de la transformation des ordres normatifs, chez les deux auteurs, à partir du sacré. L'analyse wébérienne sur ce terrain, souvent négligée par les chercheurs, permet d'intégrer les acquis de la conception durkheimienne, en diversifiant les facteurs de stabilisation normative, et en reliant le sacré au processus de "désenchantement". Les deux approches souffrent pourtant des limites d'une philosophie de la conscience d'inspiration néokantienne, qui devrait être révisée à partir d'un point de vue intersubjectif.

\section{Abstract}

The article tries to put forward the first elements of a theory of the social efficiency of norms, by using Durkheim's conception of the sacred. Starting from the sacred allows the author to reconstruct the concept of norms in Durkheim and Weber, as well as the explanation of the transformation of normative orders in both authors. Often neglected by scholars, Weber's analysis in this field makes it possible to integrate Durkheim's conception, by diversifying the factors of normative stabilization and by linking the sacred to the process of "disenchantment". However, both Weber's and Durkheim's approach suffer from the limits of a neo-Kantian philosophy of consciousness, which should be revised from an inter-subjective point of view.

\section{Resumen}

El ensayo intenta echar los cimientos de una teoría sobre la eficacia social de las normas, basándose en la concepción durkheimiana de lo sagrado. Se reconstruye de tal forma el concepto de norma en Durkheim y Weber, y la explicación de la transformación de los órdenes normativos para los dos autores, a partir de lo sagrado. El análisis weberiano sobre ese terreno, a menudo descuidado por los investigadores, permite integrar las adquisiciones de la concepción durkheimiana, diferenciando los factores de estabilización normativa, y poniendo en relación lo sagrado con el proceso de " desencantamiento ». Las dos perspectivas sufren, sin embargo, de los límites de una filosofía de la conciencia de inspiración neokantiana, que habría que revisar a partir de un punto de vista intersubjetivo. 